\title{
Nailfold Capillaroscopy in Primary Biliary Cirrhosis: a Useful Tool for the Early Diagnosis of Scleroderma
}

\author{
Francesco Tovoli ${ }^{1}$, Alessandro Granito ${ }^{1}$, Luca Giampaolo ${ }^{1}$, Magda Frisoni ${ }^{2}$, Umberto Volta ${ }^{1}$, Marco Fusconi ${ }^{2}$, Chiara Masi ${ }^{1}$, \\ Marco Lenzi ${ }^{1}$
}

1) Department of Medical and

Surgical Sciences, University

of Bologna;

2) Department of Internal

Medicine and Digestive

Diseases, Azienda

Ospedaliero-Universitaria

S.Orsola-Malpighi

Bologna, Italy

\author{
Address for correspondence: \\ Francesco Tovoli \\ Department of Medical and \\ Surgical Sciences \\ University of Bologna \\ via Massarenti 9, \\ 40138 Bologna, Italy. \\ francesco.tovoli@ \\ studio.unibo.it
}

Received: 11.11.2013

Accepted: 13.01.2014

\begin{abstract}
Background \& Aim. Primary biliary cirrhosis (PBC) is frequently associated with other autoimmune diseases, including systemic sclerosis (SSc). In the last years many efforts have been dedicated to the research of widely accepted criteria for the early diagnosis of SSc. Since studies on the prevalence of early SSc in PBC patients are lacking, our aim was to investigate its hitherto unknown prevalence in a large cohort of PBC patients. Methods. We studied 80 PBC patients and 72 patients with other chronic liver diseases. Diagnostic workup included research into signs of connective tissue disease, determination of autoantibody profile, and examination of capillary abnormalities through nailfold videocapillaroscopy.

Results. Ten PBC patients (12.5\%) satisfied diagnostic criteria for early SSc and 5 (6.3\%) had definite SSc. None of the patients in the control group were diagnosed either with early or definite SSc. No differences were observed in terms of aminotransferases, alkaline phosphatase, and liver function tests between $\mathrm{PBC}$ patients with and without associated SSc.

Conclusions. Early SSc is significantly frequent in PBC patients. The detection of early SSc in PBC patients may lead to a prompt treatment of its complications, preventing inabilities and preserving the chance of liver transplantation.
\end{abstract}

Key words: primary biliary cirrhosis - systemic sclerosis - Raynaud phenomenon - nailfold capillaroscopyanti-centromere antibodies.

Abbreviations: ACA: anti-centromere antibodies; ACR: American College of Rheumatology; AMA: antimitochondrial antibodies; ANA: anti-nuclear antibodies; CENP-B: Centromere-protein B; ENA: extractable nuclear antigens; IIF: indirect immunofluorescence; NCM: nailfold capillary microscopy; PBC: Primary biliary cirrhosis; RP: Raynaud's phenomenon; SSc: Systemic sclerosis; UDCA: ursodeoxycholic acid.

\section{INTRODUCTION}

Primary biliary cirrhosis $(\mathrm{PBC})$ is a chronic cholestatic liver disease characterized by the presence of anti-mitochondrial antibodies (AMA), abnormalities of cholestatic liver enzymes, and a variable clinical course, potentially leading to cirrhosis and liver failure [1]. Its role as a prototypic borderline and overlap disease between autoimmune hepatology and rheumatology is also well known [2-4].

Systemic sclerosis (SSc) represents the most frequent autoimmune rheumatic disease associated with $\mathrm{PBC}$, with a prevalence ranging from $5 \%$ to $12 \%[5,6]$.

Until now, prevalence of SSc in PBC populations has been evaluated according to the American College of Rheumatology (ACR) 1980 criteria [7]. These criteria, although widely validated and accepted, are characterized by a very low sensitivity. Therefore, their use implies that the diagnosis of SSc and consequently the appropriate therapy is delayed until the appearance of skin involvement and/or clinically detectable internal organ involvement $[8,9]$.

To overcome the limitation of the ACR criteria, in 2001, LeRoy and Medsger formulated criteria for an early diagnosis of the limited form of SSc. According to the criteria, patients with early SSc must have Raynaud's phenomenon (RP) plus scleroderma-type nailfold capillary changes and/or SSc-specific autoantibodies [10]. In 2008, LeRoy and Medgser criteria were validated by Koenig et al [11], and since then, they have become a routine tool used in clinical practice. 
Accordingly, in a recent review, Rigamonti et al [12] proposed an algorithm for the screening and diagnosis of SSc in patients with established $\mathrm{PBC}$, encouraging the detection of SSc-specific antibodies and the use of nailfold capillary microscopy (NCM). However, studies describing the prevalence of early SSc in PBC patients are lacking.

An early diagnosis of SSc could allow a proper treatment before the onset of severe organ damage, which could compromise the quality of life and the chance of liver transplantation.

The aim of our study was to evaluate for the first time the prevalence of early SSc in PBC patients.

\section{METHODS}

\section{Study population}

Eighty consecutive PBC patients from our Institution's Hepatology outpatient clinic were prospectively evaluated between January 2010 and December 2012.

Diagnosis of $\mathrm{PBC}$ was based on the presence of at least two of the following criteria: (i) AMA positivity by immunoenzymatic test, (ii) elevated serum levels of alkaline phosphatase for more than 6 months in the absence of an alternative explanation and (iii) typical duct lesion at histology [1]. All the PBC patients were evaluated during their follow-up and were receiving ursodeoxycholic acid (UDCA) as medication for their disease at the time of recruitment.

'Response' to UDCA was defined as a decrease in AP to $<40 \%$ of pretreatment levels or normalisation at 1 year (Barcelona criteria) [13].

As controls, 72 patients from our Hepatology outpatient clinic diagnosed with chronic liver disorders other than $\mathrm{PBC}$ were studied (44 with chronic HCV hepatitis, 12 with chronic HBV hepatitis, 7 with non-alcoholic steatohepatitis, 3 with type-1 autoimmune hepatitis, 2 with alcoholic hepatitis, 1 with Wilson disease, 1 with cardiogenic cirrhosis, and 2 with cryptogenetic chronic liver disease). All controls had a history of elevated aminotransferases over a period $>6$ months and other clinical, laboratory, or imaging features consistent with chronic liver disease. The presence of hepatitis B surface antigen or anti-HCV antibodies plus a positive HCV-RNA test permitted the diagnosis of viral hepatitis. An alcohol intake $>60 \mathrm{~g}$ per day in males and $>20 \mathrm{~g}$ per day in females had been referred by all patients diagnosed with alcoholic hepatitis. Autoimmune hepatitis was defined according to the current criteria [14]. Diagnosis of Wilson's disease was based on the recommendation by Roberts and Schilsky [15]. Non-alcoholic steatohepatitis was diagnosed on the basis of an imaging study with evidence of liver steatosis, liver biopsy specimen with evidence of fat with or without inflammation or fibrosis and history of minimal or no alcohol intake, and negative test results for viral hepatitis, autoimmune disease, and congenital liver disease [16].

Cardiac cirrhosis was defined as late-onset chronic liver failure in a patient with severe congestive heart failure and no alternative causes.

Chronic liver diseases not explained by the aforementioned causes or by biliary tract diseases were defined as 'cryptogenetic chronic liver diseases' [16].
Regarding medications, 10 patients with chronic HBV hepatitis were receiving treatment with antiviral drugs, all the patients with type 1 autoimmune hepatitis were receiving low-dose glucocorticoid drugs ( $<5 \mathrm{mg}$ of prednisone/day).

\section{Clinical evaluation}

Medical history was obtained, and a physical examination was performed according to a standard protocol. All the patients were carefully examined for the presence of physical signs including puffy fingers, digital ulcers, pitting scars, loss of digital finger pad, clinically visible capillary telangiectasias, calcinosis, and arthritis.

The presence of Raynaud's phenomenon (RP) was confirmed if patients fulfilled LeRoy and Medgser's criteria, that is, if a cold challenge induced bilateral, episodic, bi- or tri-phasic (pallor followed by dusky blueness and/or redness) colour changes of fingers [17].

\section{Autoantibodies detection}

All patients were tested for anti-nuclear antibodies (ANA) by indirect immunofluorescence (IIF) using the commercially available HEp-2 cell lines (Kallested, Chaska, MN, USA) as previously described $[18,19]$. PBC patients were also tested for anti-ENA antibodies by immunoblot assay, using EUROLINE ANA Profile 3 (Euroimmun, Lubeck, Germany), which allows the detection of IgG antibodies to each of the following recombinant antigens nRNP/Sm, Sm, SS-A/Ro-52 kD, SS-A/Ro-60 kD, SS-B/La, Scl-70, PM-Scl, Jo1, PCNA, dsDNA, Nucleosomes, Histone, Rib. P Protein, and AMA. Centromere-protein $\mathrm{B}$ (CENP-B), the major target antigen recognised by anti-centromere antibodies (ACA) pattern, is included as an additional antigen in this assay. The test was performed according to the manufacturer's instructions.

\section{Nailfold capillaroscopy}

Patients underwent a full nailfold videocapillaroscopic examination performed by a blinded examiner. All fingers apart from both thumbs were examined with a videomicroscope VideoCap 200 (DS Medigroup, Milan, Italy) at 200x magnification; traumatized or low-skin transparency fingers were not considered. Participants were left for a minimum of 15 minutes at a quiet controlled temperature $\left(20-22^{\circ} \mathrm{C}\right)$ prior to the examination.

The degree of capillary enlargement on a scale of $0-3$ and capillary loss (Grades A-D) were considered. Megacapillaries (capillary enlargement $\geq 2$ ) and/or avascular areas (capillary loss grade $\geq \mathrm{C}$ ) were considered a scleroderma pattern [20].

\section{Diagnostic criteria for SSc}

Patients were classified as having early SSc if they had RP associated with scleroderma-specific antibodies (ACA, CENP-B, SCl-70, PM/Scl) and/or scleroderma pattern at NCM $[10,11]$.

Definite SSc was defined as the fulfilment of the major ACR criterion for SSc or 2 of the 3 minor criteria $[7,10,11]$. If the ACR criteria were not fulfilled, definite SSc was defined by the presence of RP, sclerodactyly, and at least a third manifestation of CREST syndrome (calcinosis, RP, oesophageal dysmotility, sclerodactyly, telangiectasias). In the absence of 
skin involvement, definite SSc was defined by the presence of $\mathrm{RP}$ plus at least one of the following manifestations: peripheral vascular manifestations (clinically visible telangiectasias or nailfold capillaries, digital ulcers or pitting scars, loss of distal finger pad) and/or SSc visceral manifestations [11].

\section{Statistical analysis}

Statistical analysis was performed using SPSS software version 16.0 (Chicago, IL, USA). According to the type and distribution of the data, the statistical significance was estimated using Student's $t$ test, Wilcoxon test, or $2 \times 2 \chi^{2}$ or Fisher's exact tests. $\mathrm{p}$ values $<0.05$ were considered to be statistically significant.

\section{RESULTS}

The clinical and immunological features of the study population are reported in Tables I and II. Association with autoimmune conditions, RP, autoantibodies, and NCM alterations were significantly more frequent in $\mathrm{PBC}$ than in control patients. None of the control patients had NCM findings consistent with either a 'scleroderma pattern' or signs defining a condition of SSc.

On the contrary, $\mathrm{PBC}$ patients showed a high prevalence of RP (22.5\%), often associated with circulating ACA and/ or 'scleroderma-pattern'. Seven patients were simultaneously positive for RP, ACA, and 'scleroderma-pattern'; 4 were positive for both RP and scleroderma-specific antibodies (ACA in 3 cases, anti-Scl-70 in 1 case), and 4 were positive for RP and 'scleroderma-pattern' (Fig. 1). Altogether, 15 PBC patients $(18.8 \%)$ satisfied the new proposed criteria for the diagnosis of SSc: 10 of them had early SSc (12.5\%) and 5 had definite SSc $(6.25 \%)$, diagnosed on the basis of sclerodactyly and telangiectasias in all cases plus digital ulcers in 1 patient and radiological signs of pulmonary fibrosis in another.

Raynaud's phenomenon was symptomatic of an underlying SSc in $83.3 \%$ of PBC patients and in none of the control patients $(\mathrm{p}<0.005)$.

Table I. Characteristics of primary biliary cirrhosis (PBC) patients and controls

\begin{tabular}{|c|c|c|c|}
\hline & $\begin{array}{c}\mathrm{PBC} \\
\text { patients } \\
(\mathrm{n}=80)\end{array}$ & $\begin{array}{l}\text { Control } \\
\text { patients } \\
(\mathrm{n}=72)\end{array}$ & $\mathrm{p}$ \\
\hline \multicolumn{4}{|l|}{ Clinical data } \\
\hline Females/males & $77 / 3$ & $35 / 37$ & $<0.001$ \\
\hline Age $\left(\right.$ years) ${ }^{*}$ & $59(23-82)$ & $60.5(23-88)$ & 0.57 \\
\hline Cirrhosis & $11(13.8 \%)$ & $9(12.5 \%)$ & 1 \\
\hline $\begin{array}{l}\text { Time since } \\
\text { diagnosis (years) }\end{array}$ & $6(0-30)$ & $7(0-20)$ & 0.66 \\
\hline \multicolumn{4}{|l|}{ Laboratory data } \\
\hline $\operatorname{ALT}(x \text { u.n.l. })^{\star}$ & $0.7(0.5-1.2)$ & $0.75(0.5-2)$ & 0.45 \\
\hline $\operatorname{ALP}(x$ u.n.l)* & $0.9(0.5-4)$ & $0.8(0.6-2.5)$ & 0.47 \\
\hline gGT (x u.n.l)* & $0.8(0.7-7.8)$ & $1(0.5-2)$ & 0.39 \\
\hline $\begin{array}{l}\text { Total bilirubin } \\
(\mathrm{mg} / \mathrm{dL})^{*}\end{array}$ & $0.75(0.6-2.9)$ & $0.65(0.5-4.6)$ & 0.78 \\
\hline
\end{tabular}

* Median (range); ALT: alanine aminotransferase; ALP: alkaline phosphatase; gGT; gamma-glutamyl transferase; u.n.l.: upper normal limit
Table II. Clinical and immunological features of primary biliary cirrhosis (PBC) and control patients

\begin{tabular}{|c|c|c|c|}
\hline & $\begin{array}{c}\mathrm{PBC} \\
\text { patients } \\
(\mathrm{n}=80)\end{array}$ & $\begin{array}{l}\text { Control } \\
\text { patients } \\
(\mathrm{n}=72)\end{array}$ & $\mathrm{p}$ \\
\hline \multicolumn{4}{|l|}{ Clinical data } \\
\hline Raynaud's phenomenon & $18(22.5 \%)$ & $4(5.6 \%)$ & 0.0047 \\
\hline $\begin{array}{l}\text { Associated autoimmune } \\
\text { diseases }\end{array}$ & $19(23.8 \%)^{*}$ & $2(2.8 \%) \S$ & 0.0001 \\
\hline \multicolumn{4}{|l|}{ Autoantibody profile } \\
\hline ANA positive & $42(52.5 \%)$ & $24(33.3 \%)$ & 0.0218 \\
\hline $\begin{array}{l}\text { ANA positive - } \\
\text { centromere pattern }\end{array}$ & $12(15 \%)$ & $1(1.4 \%)$ & 0.0027 \\
\hline anti-SS-A/Ro 52 & $16(20 \%)$ & $1(1.4 \%)$ & 0.0002 \\
\hline anti-SS-B & $2(2.5 \%)$ & $1(1.4 \%)$ & 1 \\
\hline anti-histones & $1(1.3 \%)$ & 0 & 1 \\
\hline anti-SCl-70 & $1(1.3 \%)$ & 0 & 1 \\
\hline anti-PM/Scl & 0 & 0 & - \\
\hline anti-Sm & $1(1.3 \%)$ & 0 & 1 \\
\hline anti-nRNP/Sm & $2(2.5 \%)$ & 0 & 0.4981 \\
\hline anti-dsDNA & $1(1.3 \%)$ & 0 & 1 \\
\hline anti-CENP-B & $15(18.8 \%)$ & $1(1.4 \%)$ & 0.0004 \\
\hline $\begin{array}{l}\text { Anti-centromere } \\
\text { reactivity (positive sera) }\end{array}$ & $17(21.3 \%) \dagger$ & $1(1.4 \%) \ddagger$ & $<0.0001$ \\
\hline \multicolumn{4}{|l|}{ Nailfold videocapillaroscopy } \\
\hline Scleroderma-pattern & $15(18.8 \%)$ & 0 & $<0.0001$ \\
\hline
\end{tabular}

*Autoimmune thyroid disease ( 8 cases); coeliac diseas ( 5 cases); rheumatoid arthritis (2 cases); alopecia areata (1 case); vitiligo (1 case); Wegener granulomatosis (1 case). A patient had two distinct autoimmune diseases $\S$ Autoimmune thyroid disease (2 cases)

$\dagger 11$ patients were positive both on indirect immunofluorescence and immunoblotting, 4 only on immunoblotting and 1 only on indirect immunofluorescence

$\$ 1$ patient was positive both on indirect immunofluorescence and immunoblotting

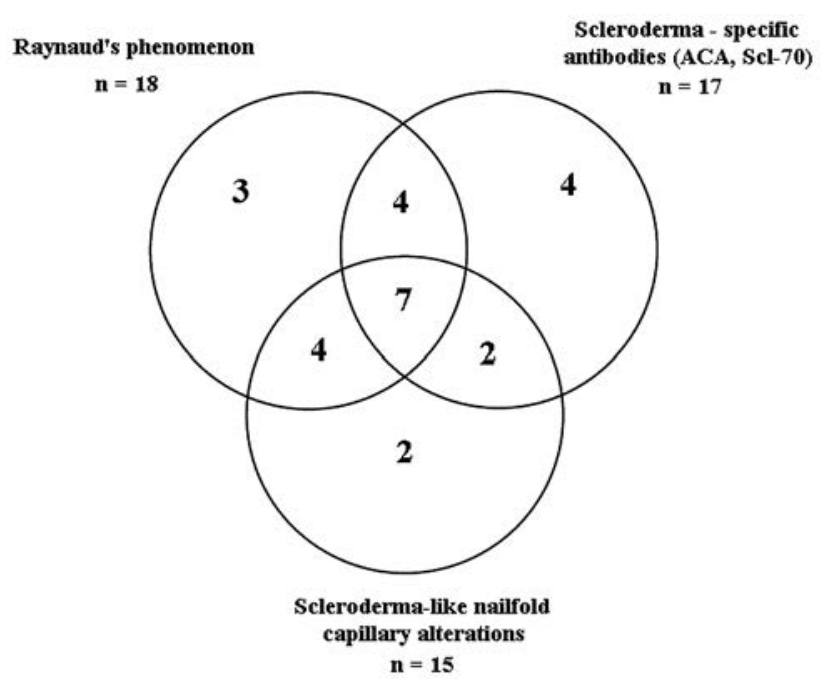

Fig. 1. Relations between Raynaud's phenomenon, scleroderma specific antibodies (anti-centromere antibodies and anti-Scl 70) and nailfold capillary alterations in patients with primary biliary cirrhosis. 


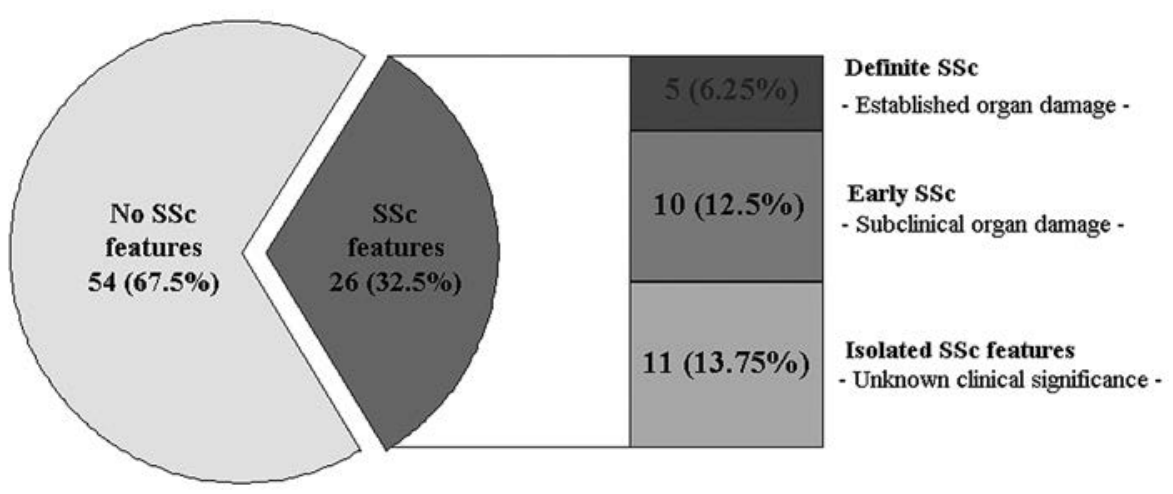

Fig. 2. Features of systemic sclerosis (SSc) as seen in our group of 80 patients with primary biliary cirrhosis. Definite and clinically evaluable, features of SSc were present only in a minority of patients. Furthermore, a quota of patients with early SSc and isolated SSc-like features could be found.

Prevalence of definite and early SSc is illustrated in Fig. 2.

Neither ACA-positivity nor scleroderma-like alterations at NCM were related to responsiveness to UDCA defined according to Barcelona criteria.

No differences were observed in terms of aminotransferases, alkaline phosphatase, and liver function tests between PBC patients with and without associated SSc (Table III). Liver cirrhosis was more frequent in patients with concurrent SSc, but this trend did not achieve a statistical significance $(\mathrm{p}=0.22)$.

Table III. Clinical and laboratory data of primary biliary cirrhosis patients with and without definite or early systemic sclerosis (SSc)

\begin{tabular}{|c|c|c|c|}
\hline & $\begin{array}{c}\text { Patients with } \\
\text { early or definite } \\
\text { SSc }\end{array}$ & $\begin{array}{c}\text { Patients } \\
\text { without SSc }\end{array}$ & $\mathrm{P}$ \\
\hline & $(n=15)$ & $(n=65)$ & \\
\hline Females/males & $15 / 0$ & $62 / 3$ & 1 \\
\hline Age $(\text { years })^{*}$ & $64(26-75)$ & $59(34-78)$ & 0.75 \\
\hline Age at diagnosis (years) ${ }^{\star}$ & $51(24-68)$ & $52(27-78)$ & 0.78 \\
\hline $\begin{array}{l}\text { Time since diagnosis } \\
\text { (years)* }\end{array}$ & $6(0-25)$ & $5(0-30)$ & 0.79 \\
\hline $\operatorname{ALT}(x \text { u.n.l })^{\star}$ & $0.7(0.5-1.2)$ & $0.75(0.5-2)$ & 0.48 \\
\hline $\operatorname{ALP}(x \text { u.n.l })^{\star}$ & $0.9(0.5-4)$ & $0.8(0.6-2.5)$ & 0.35 \\
\hline gGT (x u.n.l)* & $0.8(0.7-7.8)$ & $1(0.5-2)$ & 0.39 \\
\hline Total bilirubin $(\mathrm{mg} / \mathrm{dL})^{\star}$ & $0.75(0.6-2.9)$ & $0.65(0.5-4.6)$ & 0.76 \\
\hline $\begin{array}{l}\text { Clinical or hystological } \\
\text { cirrhosis }\end{array}$ & $4(26.7 \%)$ & $8(12.3 \%)$ & 0.23 \\
\hline
\end{tabular}

* Median (range); ALT: alanine aminotransferase; ALP: alkaline phosphatase; gGT; gamma-glutamyl transferase

\section{DISCUSSION}

It is well recognized that $\mathrm{PBC}$ overlaps not only with other chronic autoimmune liver diseases but also with other autoimmune conditions, such as Hashimoto thyroiditis, celiac disease, Sjögren syndrome, and SSc $[6,21]$.

Until now, the prevalence of SSc in PBC populations has been evaluated according to ACR 1980 criteria [7]. To our knowledge, no investigations have been performed using
LeRoy and Medsger 2001 criteria for the diagnosis of early SSc, based on the presence of documented RP associated with either scleroderma-specific autoantibodies and/or scleroderma pattern at NCM [10].

Raynaud's phenomenon, scleroderma-specific autoantibodies, and NCM alterations are known to be common features in $\mathrm{PBC}$ patients. For instance, RP prevalence has been reported to be as high as $24-30 \%$ in $\mathrm{PBC}$ patients $[5,6]$. It is also known that $\mathrm{PBC}$ patients have a high prevalence of ACA, regardless of the presence of signs or symptoms of SSc [22]. Finally, NCM alterations in PBC patients have been reported in two previous studies [23, 24], suggesting a possible role for endothelial dysfunction in the pathogenesis of PBC [23].

Our study evaluated for the first time the prevalence of early SSc in a large series of PBC patients. Our findings suggested that RP, positivity of scleroderma-specific antibodies and presence of NCM scleroderma pattern, defining a condition of early SSc, occurred in a large number of PBC patients. In particular, 15 patients (18.8\%) satisfied LeRoy and Medsger diagnostic criteria for SSc.

Only a minority of patients (6.3\% of total PBC population) had overt SSc-related organ damage, in keeping with reports in the literature $[5,6]$. This group clusters all the patients satisfying ACR 1980 criteria, with evident organ involvement and limited therapeutic possibilities to prevent further complications.

Interestingly, more patients (12.5\% of total PBC population) satisfied the criteria for early SSc. This finding is of particular interest since it has been shown that a significant percentage of early SSc patients may have a preclinical scleroderma-related heart and/or lung and/or oesophageal function abnormality, which is potentially treatable [25]. It is also known that patients diagnosed with early SSc are 60 times more likely to develop definite SSc, and possibly severe organ-based complications than the other RP patients [11].

From a therapeutic point of view, calcium channel blockers' ability to stop the disease progression is still uncertain, as well as the role of other vasoactive drugs commonly used in RP. Nevertheless, early SSc patients with lower oesophageal sphincter dysfunction should be treated with a proton pump inhibitor, and patients suffering for alveolitis should be treated with cyclophosphamide. Patients with pulmonary arterial 
hypertension can greatly benefit from specific treatments [25]. Moreover, when an anti-fibrotic agent becomes available, early SSc patients with an autoantibody profile consistent with a diffuse disease, will represent the ideal population to gain benefit from these drugs [25].

A further group of patients (13.8\% of total PBC population) was characterised by the presence of isolated SSc features, such as RP, circulating ACA, or scleroderma-like NCM alterations, confirming a sort of 'incomplete overlap' between PBC and SSc.

Little is known about the clinical significance of isolated ACA positivity and even less about NCM alterations in patients without RP. Therefore, longitudinal observation is required to clarify whether these patients are candidates for developing a scleroderma-spectrum disorder.

\section{CONCLUSIONS}

We found a high prevalence of early SSc in a large cohort of PBC patients. This finding can be of clinical relevance as an early identification of SSc may lead to an early therapeutic approach to reduce the risk of irreversible tissue and/or organ damage that may compromise a patient's quality of life and chance for liver transplantation.

We suggest that the presence of RP or ACA should trigger further investigation since they frequently herald an underlying connective tissue disease. In this setting, nailfold capillary microscopy represents a useful tool in the diagnostic workup of PBC patients to detect features suggestive for SSc, thus leading to its early diagnosis and to a prompt treatment of its major complications.

Funding and competing interests: Neither nailfold capillary microscopy nor any other technique used in this study has been sponsored/provided by the industry. The authors have no potential conflicts of interest to declare.

\section{REFERENCES}

1. Lindor KD, Gershwin ME, Poupon R, et al; American Association for Study of Liver Diseases. Primary biliary cirrhosis. Hepatology 2009;50:291-308.

2. Parveen S, Morshed SA, Nishioka M. High prevalence of antibodies to recombinant CENP-B in primary biliary cirrhosis: nuclear immunofluorescence patterns and ELISA reactivities. J Gastroenterol Hepatol 1995;10:438-445.

3. Dörner T, Held C, Trebeljahr G, Lukowsky A, Yamamoto K, Hiepe F. Serologic characteristics in primary biliary cirrhosis associated with sicca syndrome. Scand J Gastroenterol 1994;29:655-660.

4. Chou MJ, Lee SL, Chen TY, Tsay GJ. Specificity of antinuclear antibodies in primary biliary cirrhosis. Ann Rheum Dis 1995;54:148-151.

5. Marasini B, Gagetta M, Rossi V, Ferrari P. Rheumatic disorders and primary biliary cirrhosis: an appraisal of 170 Italian patients. Ann Rheum Dis 2001;60:1046-1049.

6. Watt FE, James OF, Jones DE. Patterns of autoimmunity in primary biliary cirrhosis patients and their families: a population-based cohort study. QJM 2004;97:397-406.

7. Preliminary criteria for the classification of systemic sclerosis (scleroderma). Subcommittee for scleroderma criteria of the American
Rheumatism Association Diagnostic and Therapeutic Criteria Committee. Arthritis Rheum 1980;23:581-590.

8. Ferri C, Valentini G, Cozzi F, et al; Systemic Sclerosis Study Group of the Italian Society of Rheumatology (SIR-GSSSc). Systemic sclerosis: demographic, clinical, and serologic features and survival in 1,012 Italian patients. Medicine (Baltimore) 2002;81:139-153.

9. Hunzelmann N, Genth E, Krieg T, et al. The registry of the German Network for Systemic Scleroderma: frequency of disease subsets and patterns of organ involvement. Rheumatology (Oxford) 2008;47:1185-1192.

10. LeRoy EC, Medsger TA Jr. Criteria for the classification of early systemic sclerosis. J Rheumatol 2001;28:1573-1576.

11. Koenig M, Joyal F, Fritzler MJ, et al. Autoantibodies and microvascular damage are independent predictive factors for the progression of Raynaud's phenomenon to systemic sclerosis: a twenty-year prospective study of 586 patients, with validation of proposed criteria for early systemic sclerosis. Arthritis Rheum 2008;58:3902-3912.

12. Rigamonti C, Bogdanos DP, Mytilinaiou MG, Smyk DS, Rigopoulou EI, Burroughs AK. Primary biliary cirrhosis associated with systemic sclerosis: diagnostic and clinical challenges. Int J Rheumatol 2011;2011:976427.

13. Pares A, Caballeria L, Rodes J. Excellent long-term survival in patients with primary biliary cirrhosis and biochemical response to ursodeoxycholic acid. Gastroenterology 2006;130:715-720.

14. Boberg KM, Chapman RW, Hirschfield GM, et al; International Autoimmune Hepatitis Group. Overlap syndromes: the International Autoimmune Hepatitis Group (IAIHG) position statement on a controversial issue. J Hepatol 2011;54:374-385.

15. Roberts EA, Schilsky ML; American Association for Study of Liver Diseases (AASLD). Diagnosis and treatment of Wilson disease: an update. Hepatology 2008;47:2089-2111.

16. ClarkJM, Diehl AM. Nonalcoholic fatty liver disease: an underrecognized cause of cryptogenic cirrhosis. JAMA 2003;289:3000-3004.

17. LeRoy EC, Medsger TA Jr. Raynaud's phenomenon: a proposal for classification. Clin Exp Rheumatol 1992;10:485-488.

18. Cassani F, Bianchi FB, Lenzi M, Volta U, Pisi E. Immunomorphological characterisation of antinuclear antibodies in chronic liver disease. J Clin Pathol 1985;38:801-805.

19. Granito A, Muratori P, Muratori L, et al. Antinuclear antibodies giving the ,multiple nuclear dots' or the ,rim-like/membranous' patterns: diagnostic accuracy for primary biliary cirrhosis. Aliment Pharmacol Ther 2006;24:1575-1583.

20. Maricq HR. Wide-field capillary microscopy. Arthritis Rheum 1981;24:1159-1165.

21. Granito A, Muratori P, Muratori L, et al. Antibodies to SS-A/Ro-52kD and centromere in autoimmune liver disease: a clue to diagnosis and prognosis of primary biliary cirrhosis. Aliment Pharmacol Ther 2007;26:831-838.

22. Liberal R, Grant CR, Sakkas L, Bizzaro N, Bogdanos DP. Diagnostic and clinical significance of anti-centromere antibodies in primary biliary cirrhosis. Clin Res Hepatol Gastroenterol 2013;37:572-585.

23. Marasini B, Pipia C, DeValle G, et al. Vascular impairment in patients with primary biliary cirrhosis. Int J Microcirc Clin Exp 1995;15:75-79.

24. Fonollosa V, Simeón CP, Castells L, et al. Morphologic capillary changes and manifestations of connective tissue diseases in patients with primary biliary cirrhosis. Lupus 2001;10:628-631.

25. Valentini G, Cuomo G, Abignano G, et al. Early systemic sclerosis: assessment of clinical and pre-clinical organ involvement in patients with different disease features. Rheumatology (Oxford) 2011;50:317-323. 